\title{
Chronic Kidney Disease is associated with an increase of Intimal Dendritic cells in a comparative autopsy study
}

Miguel Hueso ${ }^{1 *}$, Joan Torras', Marta Carrera ${ }^{2}$, August Vidal ${ }^{2}$, Estanis Navarro ${ }^{3+}$ and Josep Grinyó ${ }^{1+}$

\begin{abstract}
Background: Chronic Kidney Disease (CKD) and inflammation are risk factors for atherosclerotic vascular disease (ASVD). In inflammatory conditions, Nuclear Factor-KB (NF-KB) is frequently activated and it has been detected in human ASVD. In this work, we investigated if the degree of inflammation and of NF-KB activation were increased in the aorta of patients with CKD.

Methods: This is a case-control pilot study performed on 30 abdominal aorta samples from 10 human autopsies. Cases were patients with CKD and controls patients with normal glomerular filtration rate (eGFR). Infiltrating mononuclear cells $\left(\mathrm{S}_{100^{+}}, \mathrm{CD}^{+}, \mathrm{CD}^{+} \mathrm{O}^{+}, \mathrm{CD} 4 \mathrm{~L}^{+}\right)$and activation of NF-KB were identified by immunohistochemistry.

Findings: The number of cells in the intima which showed activated nuclear NF-KB correlated with severity of ASVD lesions ( $r=0.56, p=0.003$ ), with numbers of $\mathrm{CD}^{+}$lymphocytes in adventitia $(r=0.50, p=0.008)$, with numbers of CD40 ${ }^{+}$cells in the intima $(r=0.59, p=0.002)$ or in the adventitia $(r=0.45, p=0.02)$, and with numbers of $C D 40 L^{+}$ cells in the intima $(r=0.51, p=0.011)$. Increased numbers of $\mathrm{S100+}$ Intimal Dendritic cells (IDCs) were associated with ASVD $(p=0.03)$ and CKD ( $p=0.01)$.

Conclusions: Number of $\mathrm{CD}^{+}$cells, of $\mathrm{CD}_{4} \mathrm{O}^{+}$cells, of $\mathrm{CD}_{40 \mathrm{~L}}{ }^{+}$cells and the degree of NF-KB activation were increased in ASVD lesions suggesting a role for the adaptive T cell in the development of ASVD lesions. IDCs were associated both with ASVD and CKD suggesting a role of these cells in the pathogenesis of ASVD in CKD.
\end{abstract}

Keywords: Atherosclerosis, Chronic kidney disease, Inflammation, Nuclear factor-k B, Dendritic cells

\section{Findings}

Atatement of the research

The higher incidence of atherosclerotic vascular disease (ASVD) in CKD could result from systemic and/or local vascular inflammation, in which activated $\mathrm{T}$ lymphocytes would play a critical role, since their deficiency in a mouse model has been shown to lead to a marked reduction of lesions, while their adoptive transfer reconstituted the disease [1]. Activation of T lymphocytes requires a second signal provided by costimulatory molecules expressed in the membrane of antigen presenting cells (APC). CD40 and its ligand CD40L (CD154) are critical components of

\footnotetext{
* Correspondence: mhueso@idibell.cat

${ }^{\dagger}$ Equal contributors

'Department of Nephrology, Hospital Universitari Bellvitge, and Institut d'Investigació Biomèdica de Bellvitge (IDIBELL), L'Hospitalet de Llobregat, C/ Feixa llarga, s/n; L'Hospitalet de Llobregat, 08907 Barcelona, Spain Full list of author information is available at the end of the article
}

the costimulatory pathway. CD40/CD40L signalling occurs via activation of NF-kB (Nuclear Factor- $k B$ ) which activates many of the genes involved in the inflammatory response that are pivotal in the pathogenesis of vascular injury [2]. Consequently, activated NF-kB has been detected in a rabbit model of ASVD [3] and in human ASVD but not in normal vessels [4]. Furthermore, activation of $\mathrm{NF}-\mathrm{kB}$ has been also involved in the progression of renal tubulointerstitial lesions in experimental proteinuric nephropathies and in the development of human glomerulonephritis [5], a possible link between CKD and ASVD.

In this work we have studied the adaptive immune response in the aortic wall of patients with CKD, with the aim to explain the high incidence of ASVD in patients with CKD.

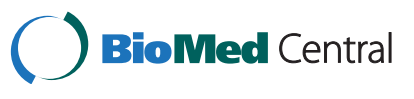

(c) 2015 Hueso et al.; licensee BioMed Central. This is an Open Access article distributed under the terms of the Creative Commons Attribution License (http://creativecommons.org/licenses/by/4.0), which permits unrestricted use, distribution, and reproduction in any medium, provided the original work is properly credited. The Creative Commons Public Domain Dedication waiver (http://creativecommons.org/publicdomain/zero/1.0/) applies to the data made available in this article, unless otherwise stated. 
Table 1 Clinical characteristics of patients

\begin{tabular}{|c|c|c|c|c|c|c|c|c|c|c|}
\hline ID & Age & Gender & Cause of death & Diabetes & Hypertension & Dyslipidemia & Creatinine $(\mu \mathrm{mol} / \mathrm{L})$ & eGFR $(\mathrm{mL} / \mathrm{min})$ & Histology & CKD stage \\
\hline 1 & 60 & Female & Cardiovascular & No & yes & No & Dialysis & & FSGS + IF-TA & $5 \mathrm{D}$ \\
\hline 2 & 83 & Female & Cardiovascular & No & yes & No & 66 & $>60$ & IF-TA & No \\
\hline 3 & 83 & Male & Cancer & Yes & Yes & No & 219 & 25 & FSGS + IF-TA & 4 \\
\hline 4 & 68 & Female & Cancer & No & No & No & 83 & $>60$ & Normal & No \\
\hline 5 & 49 & Male & Cardiovascular & No & Yes & Yes & 327 & 17 & FSGS + IF-TA & 4 \\
\hline 6 & 60 & Female & Cardiovascular & Yes & Yes & Yes & 84 & $>60$ & Normal & No \\
\hline 7 & 68 & Female & Infection & No & Yes & No & 130 & 35 & IF-TA & 3 \\
\hline 8 & 76 & Male & Infection & Yes & Yes & No & 48 & $>60$ & multicystic & No \\
\hline 9 & 86 & Female & Cardiovascular & No & Yes & No & 169 & 21 & FSGS + IF-TA & 4 \\
\hline 10 & 85 & Female & Cardiovascular & Yes & Yes & No & 66 & $>60$ & FSGS & No \\
\hline
\end{tabular}

Abbreviations:

ID: Identification Number.

eGFR: estimated Glomerular Filtration Rate.

CKD: Chronic Kidney Disease.

FSGS: focal segmental glomerulosclerosis.

IF-TA: Interticial Fibrosis and Tubular Atrophy.

\section{Methods}

\section{Study design}

This is a case-control, pilot study using aortas from patients deceased in the Hospital Universitari de Bellvitge (HUB, 11/2009-02/2010). Cases were stage 3 (or higher) CKD patients at the time of hospital admission. Controls were patients, deceased in the same time-period, with a normal eGFR (estimated glomerular filtration by the "Modification of Diet in Renal Disease" simplified equation). The presence of CKD was confirmed by histological examination.

\section{Ethics statement}

In this work, we have used authorized autopsy material from the Department of Pathology of the Hospital Universitari Bellvitge (HUB). Confidential information from patients was protected following national normatives. This manuscript has been revised by the Clinical Research Ethics Committee of the HUB.

\section{Immunohistochemistry}

Three samples were isolated from each patient: macroscopically normal abdominal aorta, incipient ASVD lesion, and a complicated lesion. Sections were H/E-stained and classified according to the modified classification of American Heart Association (AHA) [6]. Formalin-fixed, paraffinembedded aortas, were sliced at $4 \mu \mathrm{m}$ and stained with polyclonal anti-CD3, anti-S100 (Dako, Glostrup, Denmark), anti-CD40, anti-CD40L (Santa Cruz Biotechnology, Santa Cruz, CA, USA), and anti-NF-kB-p65 (phosphor S536) (Abcam, Cambridge, UK) antibodies. Sections were counterstained with hematoxylin to make nuclei evident. Human parotid glands or ganglions were used as positive controls. Isotype controls were performed using antibody buffer supplemented with irrelevant immunoglobulines of the same isotype, species and concentration as the primary antibody (ThermoFisher, Rockford, IL USA). Negative controls were performed by omitting primary antibodies. Positively stained cells were counted at x100 to x200 and acquired with a digital camera. The percentage of positive cells was assessed irrespective of the staining intensity. Results were expressed as the percentage of each population regarding the total number of cells in the intima or adventitia.

Table 2 Relative proportions of infiltrating cells in aorta samples from all patients classified according to the modified classification of AHA

\begin{tabular}{llllll}
\hline \multirow{2}{*}{ Mab } & Location & $\mathbf{n}=\mathbf{A I T}$ & $\mathbf{n}=\mathbf{P I T}$ & $\mathbf{n}=\mathbf{1 0}$ & $\mathbf{p}$ \\
\hline aCD3 & Intima & $12 \pm 9 \%$ & $20 \pm 9 \%$ & $29 \pm 6 \%^{\mathrm{t}}$ & 0.005 \\
& Adventitia & $8 \pm 6 \%$ & $21 \pm 23 \%$ & $22 \pm 14 \%$ & 0.02 \\
aS100 & Intima & $5 \pm 5 \%$ & $7 \pm 7 \%$ & $13 \pm 6 \%$ & 0.03 \\
& Adventitia & $11 \pm 9 \%$ & $9 \pm 5 \%$ & $9 \pm 3 \%$ & 0.97 \\
aCD40 & Intima & $35 \pm 20 \%$ & $69 \pm 17 \%{ }^{\mathrm{t}}$ & $73 \pm 14 \%{ }^{\mathrm{t}}$ & 0.003 \\
& Adventitia & $59 \pm 19 \%$ & $77 \pm 14 \%$ & $71 \pm 12 \%$ & 0.05 \\
aCD40L & Intima & $30 \pm 16 \%$ & $59 \pm 22 \%{ }^{*}$ & $61 \pm 25 \%{ }^{*}$ & 0.01 \\
& Adventitia & $51 \pm 15 \%$ & $52 \pm 20 \%$ & $47 \pm 13 \%$ & 0.86 \\
aNF-KB & Intima & $14 \pm 16 \%$ & $45 \pm 28 \%{ }^{*}$ & $54 \pm 25 \%{ }^{\mathrm{t}}$ & 0.01 \\
& Adventitia & $12 \pm 9 \%$ & $46 \pm 35 \%{ }^{*}$ & $40 \pm 18 \%$ & 0.01 \\
\hline
\end{tabular}

For each case we calculated the number of $\mathrm{CD}^{+}, \mathrm{S} 100^{+}, \mathrm{CD} 40^{+}, \mathrm{CD} 4 \mathrm{~L}^{+}$and $\mathrm{NF}-\mathrm{KB}^{+}$infiltrating cells as percentage of total cells and expressed the result as the mean $\pm S D$.

${ }^{*} \mathrm{P}<0.05$ vs AIT (Bonferroni Test); ${ }^{\mathrm{t} P}<0.01$ vs AIT (Bonferroni test) Abbreviations:

AIT: adaptive intimal thickening,

PIT: pathological intimal thickening,

FA: fibroatheroma. 


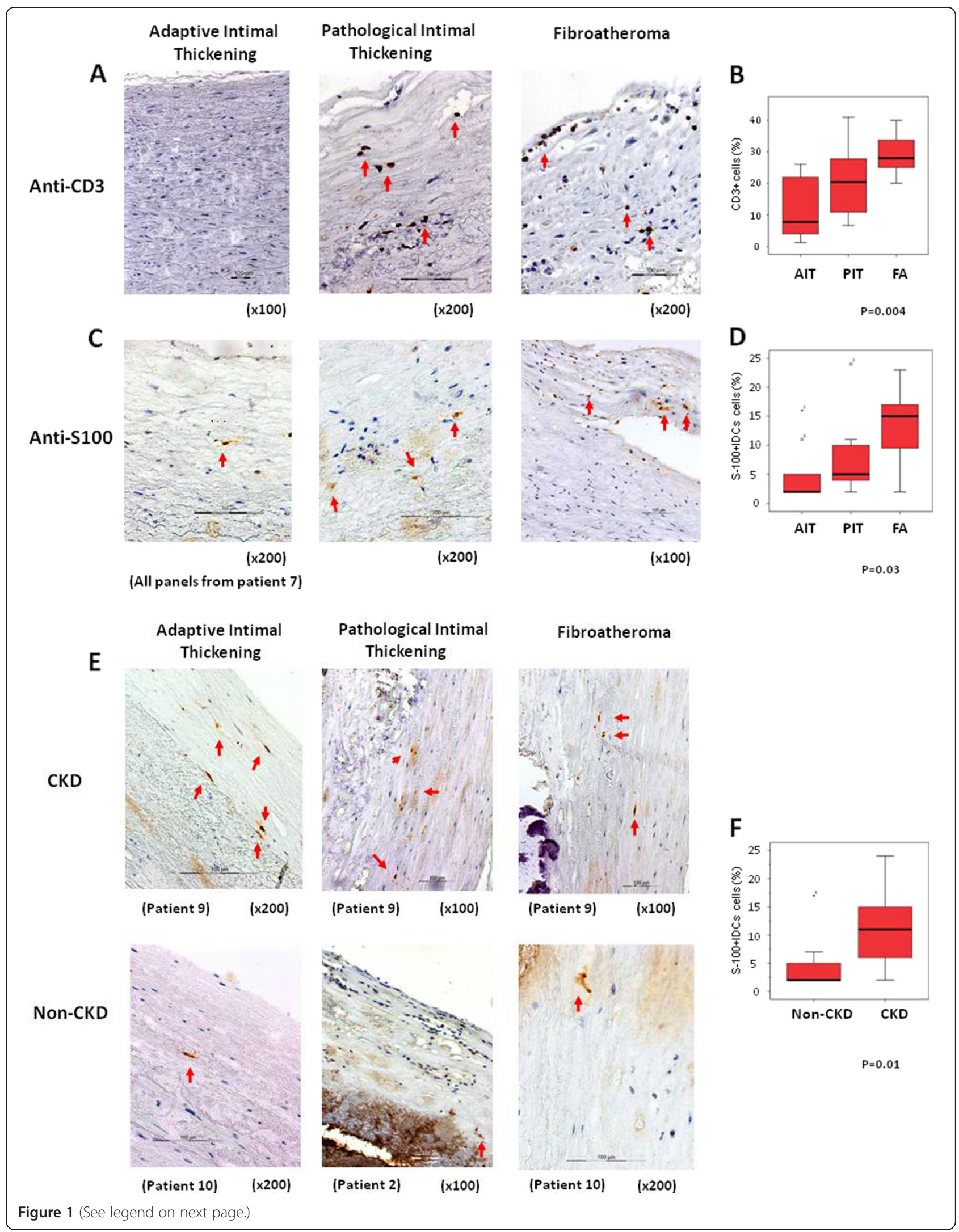


(See figure on previous page.)

Figure 1 Increased number of CD3+ T-lymphocytes and IDCs in areas of progressive ASVD lesions. A. CD3+ T-lymphocytes detected in the endothelium and subendothelial zones of aortas with PIT or FA (arrows). Magnifications as indicated. B. Box-plot showing percentage of CD3 + T-lymphocytes, regarding the total number of cells in the field, according to histological lesion. Kruskal-Wallis test. C. S100 ${ }^{+}$IDCs, localized in the intima of aortas according to histological lesion. Kruskal-Wallis test. Magnifications as indicated. D. Box-plot showing percentage of S-100 ${ }^{+}$DCS according to histological lesion. Kruskal-Wallis test. E. S100+ IDCs, localized in the intima of aortas grouped according to histological lesions and presence of CKD. Magnifications as indicated. F. Box-plot showing percentage of S100 ${ }^{+}$IDCs aCCording to CKD and with independence of histological lesion. Mann-Whitney test. In figures B, D and F, each diagram represents the median, quartiles and outliers. The colored box represents the interquartile range that contains $50 \%$ of the values. The whiskers are lines that extended from the box to the highest and lowest values, excluding outliers. A line across the box represents the median value.

\section{Statistical analysis}

Cell infiltration was measured as the mean of scores \pm standard deviation. Differences in cell infiltration between arterial lesions were compared by the Kruskal-Wallis test. Mann-Whitney test was used to compare differences in cell infiltration in CKD patients. Correlation among CD3, S100, CD40, CD40L and NF- $\mathrm{BB}$ scores in ASVD lesions against the presence of CKD was performed with the Spearman rank correlation coefficient. A $\mathrm{p}<0.05$ value was considered statistically significant. $\mathrm{P}$ values were corrected for the number of variables compared according to the Bonferroni method. Statistical analysis was performed using the SPSS 12.0 software (SPSS Inc. Chicago, IL).

\section{Results and discussion}

Atherosclerotic vascular disease (ASVD) is more common and more severe in CKD patients than in the general population $[1,7]$ and ASVD lesions grow faster in an uremic environment [8] suggesting a pathogenic link. Since inflammation seems critical in the development of ASVD pathogenesis [9], the higher incidence of ASVD in CKD could result from systemic and/or local vascular inflammation. Thus, we present a pilot study in 30 samples from abdominal aortas, obtained from 10 deceased patients (Table 1) to investigate whether the abdominal aorta from CKD patients presented features of enhanced inflammation that would justify the increase in ASVD lesions observed in these patients.

A few infiltrating $\mathrm{CD}^{+}$cells were found in the intima of aortas with adaptive intimal thickening (AIT) with their number increasing significantly in areas with pathological intimal thickening (PIT) or with fibroatheroma (FA) (Table 2), and correlating with the severity of the ASVD lesions (Figure 1A and B). These results are not surprising since inflammation seems to have a critical role in ASVD pathogenesis [9], subsequent to the development of maladaptive vascular inflammation as well as to the failure of

Table 3 Relative proportions of infiltrating cells in aorta samples grouped according to presence of CKD and to the modified classification of American Heart Association (AHA)

\begin{tabular}{|c|c|c|c|c|c|c|c|c|c|}
\hline \multirow[b]{2}{*}{ Mab } & \multirow[b]{2}{*}{ Location } & \multicolumn{3}{|c|}{ Non-CKD } & \multicolumn{4}{|c|}{ CKD } & \\
\hline & & $\begin{array}{l}\text { AlT } \\
n=5\end{array}$ & $\begin{array}{l}\text { PIT } \\
n=5\end{array}$ & $\begin{array}{l}\mathrm{FA} \\
\mathrm{n}=5\end{array}$ & $\mathbf{p}$ & & $\begin{array}{l}\text { PIT } \\
n=5\end{array}$ & $\begin{array}{l}\mathrm{FA} \\
\mathrm{n}=5\end{array}$ & $\mathbf{p}$ \\
\hline \multirow[t]{2}{*}{ aCD3 } & intima & $10 \pm 9 \%$ & $18 \pm 10 \%$ & $25 \pm 5 \%$ & 0.05 & $14 \pm 12 \%$ & $23 \pm 11 \%$ & $33 \pm 6 \%$ & 0.06 \\
\hline & adventitia & $8 \pm 5 \%$ & $11 \pm 5 \%$ & $22 \pm 11 \%$ & 0.14 & $7 \pm 8 \%$ & $31 \pm 31 \%$ & $23 \pm 18 \%$ & 0.07 \\
\hline \multirow[t]{2}{*}{ aS100 } & intima & $3 \pm 1 \%$ & $3 \pm 1 \%$ & $11 \pm 7 \%$ & 0.11 & $8 \pm 6 \%$ & $11 \pm 8 \%$ & $16 \pm 5 \%$ & 0.22 \\
\hline & adventitia & $10 \pm 10 \%$ & $8 \pm 5 \%$ & $8 \pm 3 \%$ & 0.85 & $13 \pm 9 \%$ & $9 \pm 5 \%$ & $9 \pm 5 \%$ & 0.78 \\
\hline \multirow[t]{2}{*}{ aCD40 } & intima & $38 \pm 19 \%$ & $70 \pm 15 \% *$ & $84 \pm 6 \%{ }^{t}$ & 0.01 & $30 \pm 26 \%$ & $66 \pm 25 \%$ & $65 \pm 12 \%$ & 0.11 \\
\hline & adventitia & $61 \pm 10 \%$ & $69 \pm 15 \%$ & $76 \pm 6 \%$ & 0.16 & $57 \pm 29 \%$ & $86 \pm 7 \%$ & $66 \pm 16 \%$ & 0.07 \\
\hline \multirow[t]{2}{*}{ aCD40L } & intima & $28 \pm 18 \%$ & $51 \pm 22 \%$ & $69 \pm 31 \%$ & 0.08 & $35 \pm 14 \%$ & $70 \pm 20 \%$ & $51 \pm 15 \%$ & 0.06 \\
\hline & adventitia & $46 \pm 16 \%$ & $40 \pm 15 \%$ & $53 \pm 14 \%$ & 0.46 & $58 \pm 12 \%$ & $68 \pm 16 \%$ & $40 \pm 9 \%$ & 0.09 \\
\hline \multirow[t]{2}{*}{ aNF-kB } & intima & $10 \pm 18 \%$ & $49 \pm 30 \%$ & $46 \pm 17 \%$ & 0.07 & $19 \pm 12 \%$ & $42 \pm 29 \%$ & $62 \pm 32 \%$ & 0.11 \\
\hline & adventitia & $11 \pm 11 \%$ & $58 \pm 35 \% *$ & $31 \pm 6 \%$ & 0.03 & $14 \pm 9 \%$ & $31 \pm 32 \%$ & $62 \pm 18 \%$ & 0.22 \\
\hline
\end{tabular}

For each case we calculated the number of $\mathrm{CD}^{+}, \mathrm{S} 00^{+}, \mathrm{CD} 40^{+}, \mathrm{CD}_{40} \mathrm{~L}^{+}$and $\mathrm{NF}-\mathrm{KB}^{+}$infiltrating cells as percentage of total cells and expressed the result as the mean \pm SD.

${ }^{*} \mathrm{P}<0.05$ vs AIT (Bonferroni Test); ${ }^{\mathrm{t} P}<0.005$ vs AIT (Bonferroni test).

Abbreviations:

AIT: adaptive intimal thickening,

PIT: pathological intimal thickening,

FA: fibroatheroma.

CKD: Chronic kidney Disease 


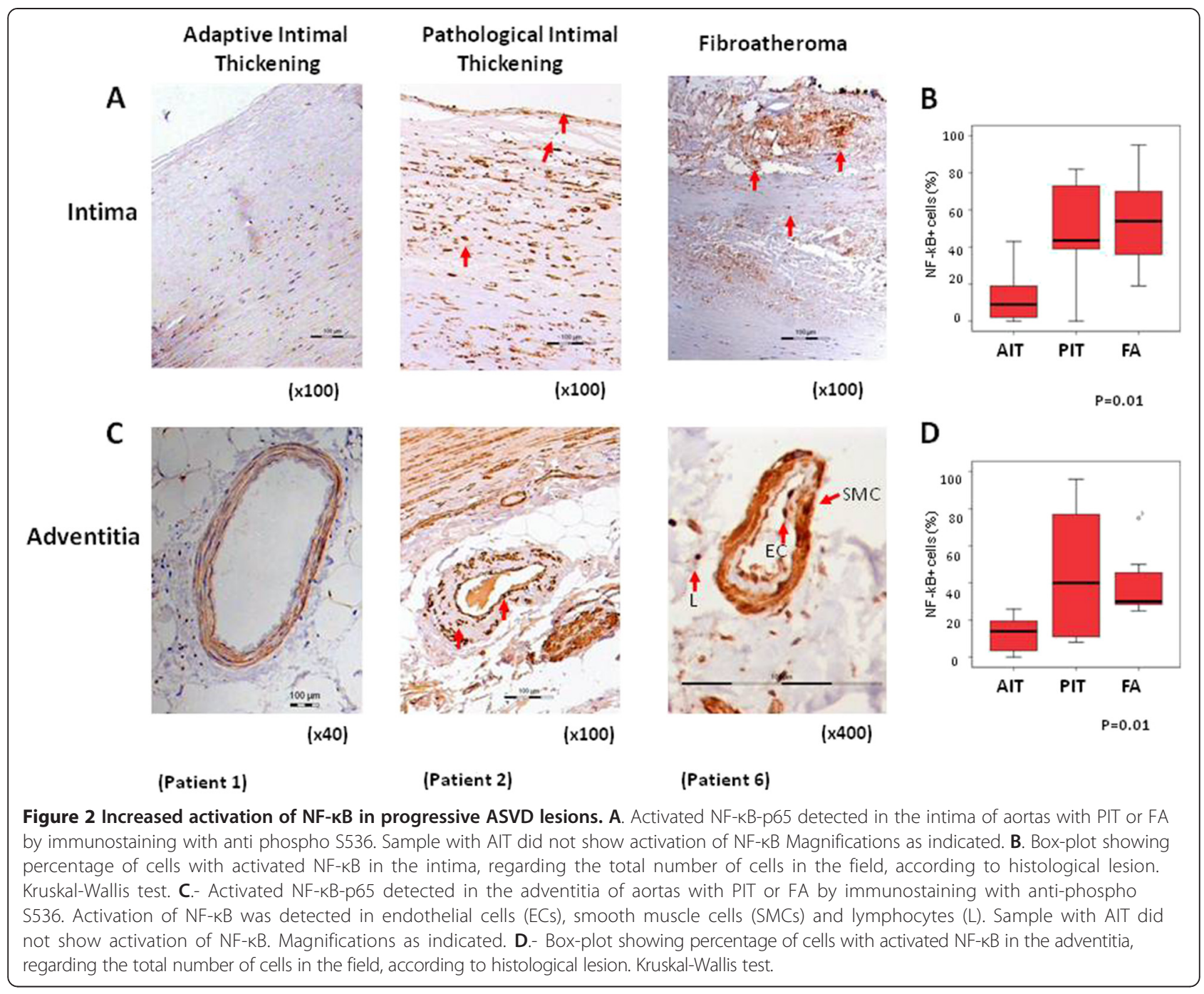

inflammation-resolving regulatory processes in response to the deposition and modification of low-density lipoproteins within arterial walls [10].

Since Dendritic Cells (DCs) are key sensors of the innate and the adaptive arms of the immune system, we analyzed their presence in the aortic wall. There are several markers for the identification of DCs but it has been suggested that S100 is the most reliable marker for the visualization of the major portion of $\mathrm{DC}$ population in the human arterial intima [11]. Although S100 is not exclusively specific to DC and it is expressed by neural cells, S100 specifically identifies DC in the arterial intima because the intima and two-thirds of the internal part of the tunica media are not innervated [12]. The proportion of $\mathrm{S} 00^{+}$Intimal Dendritic Cells (IDCs) was also increased in progressive ASVD lesions (Table 2 and Figure 1C) and in patients with CKD $(11 \pm 6 \%$ of IDCs in CKD aortas vs. $5 \pm 5 \%$ in aortas without CKD, $\mathrm{p}=0.01$; Figure $1 \mathrm{E}$ and $\mathrm{F}$ ), suggesting a role of these cells in the pathogenesis of ASVD in CKD. Other works have detected IDCs in atherogenic susceptible regions in the aorta of rabbits and mice, in which their abundance was correlated with a genetic susceptibility of mice to ASVD [13]. On the contrary depletion of IDCs was shown to reduce intimal lipid accumulation, indicating that IDCs would be involved in the formation of nascent atherosclerotic lesions [14]. Furthermore, it has been suggested that a proinflammatory environment, such as CKD, might promote IDCs accumulation and predispose these regions to ASVD [13]. The origin of IDCs and the physiological role of IDCs in the normal aorta is still unknown. IDCs isolated from the aorta and valves of mice have the capacity to crosspresent antigen to $\mathrm{CD}^{+} \mathrm{T}$ cells in vitro, but the scarcity of T-cells in the aortic intima suggests that antigen presentation would not be their primary function. In this sense, it has been recently suggested that initial foam cells in early lesions would derive primarily from IDCs rather than from newly recruited monocytes [13].

AIT lesions showed scanty $\mathrm{CD}_{4} 0^{+}$-expressing cells in the intima with their numbers increasing in areas with 
progressive ASVD lesions as well as in the vessels of the adventitia according to the severity of the lesions (Table 2). In addition, the proportion of $\mathrm{CD}_{40 \mathrm{~L}^{+}}$infiltrating cells was increased in progressive ASVD lesions (Table 2) and $\mathrm{CD}_{40 \mathrm{~L}^{+}}$expression was induced in endothelial cells of vasa vasorum suggesting an endothelial activation. These data are not surprising because the CD40/ CD40L system has been already implicated in ASVD pathogenesis [15]. However, no relationship could be established with CKD in this work (Table 3).

Since NF-kB is a downstream target of CD40/CD40L signalling we analysed NF- $\mathrm{kB}$ activation in the progressive ASVD lesions, as well as its association with the accumulation of inflammatory cells, by using an antibody specific for the phospho-S536 form of NF-kB-p65 (see Methods). Activation of NF- $\mathrm{kB}$ was detected in endothelial cells (ECs), smooth muscle cells (SMCs) and lymphocytes (Figure 2A, second and third panels). Furthermore, numbers of activated NF- $\mathrm{KB}^{+}$cells in the intima correlated positively with the severity of ASVD lesions $(r=0.56, p=$ 0.003 ) (Figure 2), and with the percentage of $\mathrm{CD}^{+}$cells in the adventitia $(r=0.50, p=0.008)$, of $C D 40^{+}$cells $(r=0.59$, $\mathrm{p}=0.002$ in the intima, and $\mathrm{r}=0.45, \mathrm{p}=0.02$ in the adventitia), and of CD40L $\mathrm{L}^{+}$cells $(\mathrm{r}=0.44, \mathrm{p}=0.03$ in the intima). No differences were detected in the number of activated $\mathrm{NF}-\mathrm{KB}^{+}$cells in aortas from CKD patients vs. controls without CKD.

In summary, our study showed an accumulation of infiltrated $\mathrm{CD}^{+}$lymphocytes, of $\mathrm{CD} 40^{+}$cells as well as an increase in the activation of NF- $\mathrm{kB}$ associated to progressive ASVD lesions in the abdominal aorta. The arterial wall of abdominal aorta displayed an increase number of intimal dendritic cells (IDCs) in patients with CKD suggesting a role of these cells in the pathogenesis of ASVD in CKD.

\section{Abbreviations}

ASVD: Atherosclerotic Vascular Disease; CKD: Chronic Kidney Disease; AIT: Adaptive Intimal Thickening; PIT: Pathological Intimal Thickening; FA: Fibroatheroma; IDCs: Intimal Dendritic Cells; aNF-KB: Activated Nuclear Factor-KB.

\section{Competing interests}

The authors declare that have no competing interests.

\begin{abstract}
Authors' contributions
The contribution of each authors were as follows: MH conceived, designed, performed experimental work, analyzed, interpreted data and wrote the paper; JT conceived, designed and interpreted data; $M C$ and $A V$ : performed experimental work, analyzed and interpreted data; EN analyzed, interpreted data and wrote the paper, and JG provided intellectual content of critical importance to the work and contributed with reagents, materials and analysis tools. All authors read and approved the final manuscript.
\end{abstract}

\section{Acknowledgments}

We acknowledge the excellent technical assistance of Mar Varela from Biobanc HUB-ICO-IDIBELL, and Nuria Bolaños and Christian Varela from Laboratori de Nefrologia Experimental-IDIBELL.

This work was supported by Fondo de Investigaciones Sanitarias, Ministerio de Sanidad y Consumo, (PI11/00556) to MH, REDinREN (12/0021) and by the
Xarxa de Bancs de Tumors de Catalunya sponsored by Pla Director d'Oncología de Catalunya (XBTC).

\section{Author details}

'Department of Nephrology, Hospital Universitari Bellvitge, and Institut d'Investigació Biomèdica de Bellvitge (IDIBELL), L'Hospitalet de Llobregat, C/ Feixa llarga, s/n; L'Hospitalet de Llobregat, 08907 Barcelona, Spain. ${ }^{2}$ Department of Pathology, Hospital Universitari Bellvitge, Barcelona, Spain. 'aboratori d'Oncologia Molecular, Institut d'Investigació Biomèdica de Bellvitge (IDIBELL), L'Hospitalet de Llobregat, Spain.

Received: 9 January 2015 Accepted: 20 March 2015

Published online: 29 March 2015

\section{References}

1. London GM, Guerin AP, Marchais SJ, Métivier F, Pannier B, Adda H. Arterial media calcification in end-stage renal disease: impact on all-cause and cardiovascular mortality. Nephrol Dial Transplant. 2003;18:1731-40.

2. Collins T, Cybulsky MI. NF-kappaB: pivotal mediator or innocent bystander in atherogenesis? J Clin Invest. 2001;107:255-64.

3. Hernandez-Presa M, Bustos C, Ortego M, Tuñon J, Renedo G, Ruiz-Ortega M. Angiotensin-converting enzyme inhibition prevents arterial nuclear factor-kappa $B$ activation, monocyte chemoattractant protein-1 expression, and macrophage infiltration in a rabbit model of early accelerated atherosclerosis. Circulation. 1997;95:1532-41.

4. Zhang W, Xing SS, Sun XL, Xing QC. Overexpression of activated nuclear factor-kappa B in aorta of patients with coronary atherosclerosis. Clin Cardiol. 2009;32:E42-7.

5. Zheng L, Sinniah R, Hsu SI. Pathogenic role of NF-kappaB activation in tubulointerstitial inflammatory lesions in human lupus nephritis. J Histochem Cytochem. 2008;56:517-29.

6. Virmani R, Kolodgie FD, Burke AP, Farb A, Schwartz SM. Lessons from sudden coronary death: a comprehensive morphological classification scheme for atherosclerotic lesions. Arterioscler Thromb Vasc Biol. 2000;20:1262-75.

7. Go AS, Chertow GM, Fan D, McCulloch CE, Hsu CY. Chronic kidney disease and the risks of death, cardiovascular events, and hospitalization. N Engl J Med. 2004;351:1296-305.

8. Massy ZA, Ivanovski O, Nguyen-Khoa T, Angulo J, Szumilak D, Mothu N. Uremia accelerates both atherosclerosis and arterial calcification in apolipoprotein E knockout mice. J Am Soc Nephrol. 2005;16:109-16.

9. Libby P, Ridker PM, Maseri A. Inflammation and atherosclerosis. Circulation. 2002;105:1135-43.

10. Alberts-Grill N, Denning TL, Rezvan A, Jo H. The role of the vascular dendritic cell network in atherosclerosis. Am J Physiol Cell Physiol. 2013;305:C1-C21.

11. Bobryshev YV. Dendritic cells and their role in atherogenesis. Lab Invest. 2010;90:970-84.

12. Bobryshev YV, Lord RS. S-100 positive cells in human arterial intima and in atherosclerotic lesions. Cardiovasc Res. 1995;29:689-96.

13. Cybulsky MI, Jongstra-Bilen J. Resident intimal dendritic cells and the initiation of atherosclerosis. Curr Opin Lipidol. 2010;21:397-403.

14. Jung S, Unutmaz D, Wong P, Sano G, De los Santos K, Sparwasser T. In vivo depletion of $C D 11 \mathrm{C}+$ dendritic cells abrogates priming of $C D 8+T$ cells by exogenous cell-associated antigens. Immunity. 2002;17:211-20.

15. Packard RR, Libby P. Inflammation in atherosclerosis: from vascular biology to biomarker discovery and risk prediction. Clin Chem. 2008;54:24-38. 